\title{
DETERMINAÇ̃̃O DA SOLUÇÃO ÓTIMA DO PROBLEMA DE FLUXO DE POTẾNCIA ÓTIMO VIA ANÁLISE DE SENSIBILIDADE
}

\author{
Edmarcio Antonio Belati * \\ Alessandra Macedo de Souza \\ Universidade de São Paulo (USP) \\ São Carlos - SP \\ belati@sel.eesc.usp.br; amacedo@sel.eesc.usp.br \\ Edméa Cássia Baptista \\ Universidade Estadual Paulista (UNESP) \\ Bauru - SP \\ $\underline{\text { baptista@ffc.unesp.br }}$ \\ Geraldo R. M. da Costa \\ Universidade de São Paulo (USP) \\ São Carlos - SP \\ geraldo@sel.eesc.usp.br \\ * Corresponding author / autor para quem as correspondências devem ser encaminhadas \\ Recebido em 10/2004; aceito em 07/2005 após 1 revisão \\ Received October 2004; accepted July 2005 after one revision
}

\section{Resumo}

Neste trabalho propomos uma abordagem para a resolução do problema de Fluxo de Potência Ótimo (FPO) perturbado. A metodologia consiste na utilização de análise de sensibilidade para estimar novas soluções depois de ocorridas algumas perturbações no problema a partir de uma solução ótima obtida via um programa de FPO. Estas perturbações podem ser variações de carga em uma ou mais barras do sistema. A técnica de análise de sensibilidade é baseada nas informações de segunda ordem e nas condições de Karush-Kuhn-Tucker (KKT). A obtenção da solução após ocorrerem perturbações no sistema é direta e não necessita de parâmetros iniciais e de correção como os de penalidade e de barreira, utilizados nos programas de FPO convencionais. Os resultados numéricos apresentados evidenciam o potencial desta metodologia para resolução do problema de FPO para pequenas perturbações.

Palavras-chave: análise de sensibilidade; fluxo de potência ótimo; perturbação; condições de Karush-Kuhn-Tucker.

\begin{abstract}
We propose in this work an approach for solving the perturbated Optimal Power Flow (OPF) problem. The methodology consists in the use of sensitivity analysis to estimate new solutions after occurring some perturbations in the problem starting from optimal solution obtained via an OPF program. These perturbations consist in loading variations in some busses of the system. The sensitivity analysis technique is based on the information of second order and in the Karush-Kuhn-Tucker conditions. The obtaining of the solutions after the occurrence of perturbations in the system is direct and it doesn't need initial parameters and correction parameters as other methods like penalty and barrier used in the conventional OPF programs need. The numerical results demonstrate the potential of this methodology for the solution of the perturbated OPF problem.
\end{abstract}

Keywords: sensitivity analysis; optimal power flow problem; perturbation; Karush-Kuhn-Tucker conditions. 


\section{Introdução}

Um Sistema Elétrico de Potência (SEP) opera em regime permanente, porém devido à flutuação nas demandas uma perturbação é introduzida. Se as variáveis de controle, após uma perturbação, ficarem constantes, isto implicará em um novo ponto de operação com tensões e ângulos alterados nas barras da rede. Essas alterações podem levar uma ou mais variáveis de estado para fora de seus limites operacionais. Para obter novamente um ponto operacional seria necessário resolver o novo problema perturbado ou exercer um controle sobre o sistema, atuando nas variáveis de controle. Se este ajuste tiver sucesso, então, as variáveis dependentes voltarão ao estado operacional, isto é, dentro de seus limites. É de fundamental importância, em um sistema de controle, o completo entendimento de como as variações no sistema afetam seu estado. Tal entendimento pode ser obtido a partir da análise de sensibilidade, sem a necessidade de se processar ou reprocessar vários casos de Fluxo de Potência Ótimo (FPO). Não só a sensibilidade de uma variável pode ser obtida em relação a uma variável qualquer, mas a sensibilidade de quaisquer variáveis controladas pode ser obtida com relação a todas as variáveis de controle e perturbações do sistema.

A análise de sensibilidade é uma exigência de várias áreas de pesquisa. Em um SEP a aplicação de análise de sensibilidade vem sendo utilizada com grande destaque sendo, amplamente, aplicada no planejamento de potência reativa (Iba et al., 1998; e Kishore et al., 1971). Os autores Belhadj et al. (1996) e Aumuller (2002) utilizaram sensibilidade no estudo de instabilidade de tensão. Park \& Lee (1997) apresentaram um estudo de sensibilidade para restauração de sistemas de potência. A idéia básica destes métodos consiste em trabalhar com a linearização das equações do problema de Fluxo de Potência (FP). A análise de sensibilidade proposta por Fiacco (1976) foi utilizada na resolução do problema de FPO por Dillon T.S. (1980), onde o objetivo era minimizar o custo de geração. Gribik et al. (1990), basearam no teorema Fiacco (1976), mas trabalharam com as restrições de desigualdade relaxadas, o que resultou em uma matriz sensibilidade incompleta.

Neste trabalho nós utilizaremos a análise de sensibilidade para determinar o novo ponto de operação para o problema de FPO depois de ocorrida alguma perturbação no sistema. A função objetivo utilizada é minimizar as perdas de potência ativa na transmissão, está função é não linear, não convexa e não separável. Este estudo de sensibilidade também permitirá a análise do sistema de maneira fácil e direta, isto é, a verificação do novo desempenho da rede (função objetivo) e a sensibilidade de uma determinada variável em relação às variações da demanda.

Este trabalho está dividido da seguinte forma: primeiramente na seção 2 será apresentada a formulação do problema de FPO e na seção 3 o desenvolvimento da metodologia de sensibilidade utilizada; em seguida, na seção 4, será mostrada a aplicação da metodologia ao problema de FPO, e realizados testes numéricos para avaliar o potencial da metodologia de sensibilidade; e na seção 5 serão apresentadas as conclusões do trabalho.

\section{Formulação do Problema de Fluxo de Potência Ótimo}

O problema FPO poder ser considerado um problema de Programação Não-Linear (PNL), e é representado matematicamente por: 


$$
\begin{array}{ll}
\min & f(x) \\
\text { s.a } & g_{i}(x)=0, i=1, \cdots, m \\
& h_{j}(x) \leq 0, j=1, \cdots, r \\
& x^{\min } \leq x \leq x^{\max }
\end{array}
$$

onde: $x^{T}=(\theta, V, t) \in R^{n}$ representa o vetor das variáveis de estado e controle do sistema, isto é, ângulos de fase, magnitudes das tensões e tap dos transformadores; $x^{\min }$ e $x^{\max }$ representam os vetores dos seus limites inferiores e superiores, respectivamente; $f(x)$ representa as perdas de potência ativa na transmissão; $g(x)=0$ representa o conjunto das equações de balanço do fluxo de potência; $h(x) \leq 0$ representa o conjunto das restrições funcionais, isto é, limites de potência ativa e reativa nas linhas de transmissão e transformadores, limites de injeção de potência reativa nas barras de controle de reativos e injeção de potência ativa na barra slack. Este é um típico problema não-linear, não convexo e de grande porte. A solução do problema (1) pode ser obtida por algum programa de FPO. Entre eles podemos destacar: Granville (1994), Costa (1997), e Baptista et al. (2004). Neste trabalho utilizamos Baptista et al. (2004).

\section{Técnica de Sensibilidade}

A técnica de análise de sensibilidade apresentada é baseada no teorema proposto por Fiacco (1976). O teorema utiliza a análise de sensibilidade de primeira ordem aplicada à solução local de segunda ordem. Essa técnica pode ser utilizada para estimar a nova solução de um problema de PNL depois de ocorridas perturbações no problema. As perturbações podem ocorrer tanto nas restrições como na função objetivo. A técnica de analise de sensibilidade apresentada nesta seção considera as perturbações nas restrições de igualdade, quando há uma variação nas restrições de igualdade (potência ativa e reativa nas barras de carga do sistema). A formulação matemática da técnica de análise de sensibilidade utilizada é apresentada a seguir.

Ao problema (1) são introduzidas perturbações, $\varepsilon_{i}, i=1, \cdots, m$, nas restrições de igualdade.

$$
\begin{array}{ll}
\min & f(x) \\
\text { s.a } & g_{i}(x)+\varepsilon_{i}=0, \quad i=1, \ldots, m \\
& h_{j}(x) \leq 0, \quad j=1, \ldots, r \\
& x^{\min } \leq x \leq x^{\max }
\end{array}
$$

em que, $\varepsilon=\left(\varepsilon_{1}, \ldots, \varepsilon_{m}\right)^{T}$ é o vetor perturbação. Associa-se a seguinte função Lagrangiana ao problema (2):

$$
L(x, \mu, \lambda, \varepsilon)=f(x)+\sum_{i=1}^{m} \lambda_{i}\left[g_{i}(x)+\varepsilon_{i}\right]+\sum_{j=1}^{r} \mu_{j} h_{j}(x)
$$

em que $\lambda$ é o vetor dos multiplicadores de Lagrange associados às restrições de igualdade, $\mathrm{e}$ $\mu$ é o vetor dos multiplicadores de Lagrange associados as restrições de desigualdade. 
Para aplicar a técnica de análise de sensibilidade, é preciso ter primeiramente a solução ótima para o problema, $\left(x^{*}, \lambda^{*}, \mu^{*}\right)$, sem perturbação, ou seja, a solução para o problema (2) com $\varepsilon=0$. Neste artigo esta solução é obtida através de um programa FPO.

A técnica de análise de sensibilidade considera o gradiente da função Lagrangiana, as condições de folgas complementares e as restrições de igualdade perturbadas, isto é:

$$
\begin{array}{ll}
\nabla_{x} L(x, \mu, \lambda, \varepsilon)=0 & \\
\mu_{j}\left[h_{j}(x)\right]=0, & j=1, \ldots, r \\
g_{i}(x)+\varepsilon_{i}=0, & i=1, \ldots, m
\end{array}
$$

em que, $\mu \geq 0$ e $\lambda$ irrestrito. O gradiente da função Lagrangiana (3) é representado por:

$$
\nabla_{x} L(x, \mu, \lambda, \varepsilon)=\nabla_{x} f(x)+\sum_{i=1}^{m} \lambda_{i} \nabla_{x} g_{i}(x)+\sum_{j=1}^{r} \mu_{j} \nabla_{x} h_{j}(x)
$$

As raízes do sistema não-linear (4) são determinadas linearizando o sistema no ponto ótimo $\left(x^{*}, \lambda^{*}, \mu^{*}\right)$, o que resulta no seguinte sistema linear:

$$
\begin{aligned}
& \nabla_{x} L\left(x^{*}, \mu^{*}, \lambda^{*}, \varepsilon\right)+\nabla_{x x}^{2} L\left(x^{*}, \mu^{*}, \lambda^{*}, \varepsilon\right) \Delta x+ \\
& +\nabla_{x \mu}^{2} L\left(x^{*}, \mu^{*}, \lambda^{*}, \varepsilon\right) \Delta \mu+\nabla_{x \lambda}^{2} L\left(x^{*}, \mu^{*}, \lambda^{*}, \varepsilon\right) \Delta \lambda=0 \\
& \mu_{j}^{*} h_{j}\left(x^{*}\right)+\mu_{j}^{*} \nabla_{x} h_{j}\left(x^{*}\right) \Delta x+h_{j}\left(x^{*}\right) \Delta \mu=0, j=1, \ldots, r \\
& {\left[g_{i}\left(x^{*}\right)+\varepsilon_{i}\right]+\nabla_{x} g_{i}\left(x^{*}\right) \Delta x=0, i=1, \ldots, m}
\end{aligned}
$$

Reescrevendo (6) e eliminando os termos nulos temos:

$$
\begin{array}{ll}
\nabla_{x x} L\left(x^{*}, \mu^{*}, \lambda^{*}, \varepsilon\right) \Delta x+\nabla_{x} h_{j}\left(x^{*}\right) \Delta \mu+\nabla_{x} g_{i}\left(x^{*}\right) \Delta \lambda & =0 \\
\mu_{j}^{*} \nabla_{x} h_{j}\left(x^{*}\right) \Delta x+h_{j}\left(x^{*}\right) \Delta \mu & =0 \\
\varepsilon_{i}+\nabla_{x} g_{i}\left(x^{*}\right) \Delta x & =0
\end{array}
$$

O conjunto das equações (7) pode ser representado na forma matricial por:

$$
\left[\begin{array}{l}
x(\varepsilon)-x^{*} \\
\mu(\varepsilon)-\mu^{*} \\
\lambda(\varepsilon)-\lambda^{*}
\end{array}\right]=-\left[\begin{array}{ccc}
\nabla^{2} L^{*} & H^{*} & G^{*} \\
\mathrm{M}^{*}\left(H^{*}\right)^{T} & D_{H}{ }^{*} & 0 \\
\left(G^{*}\right)^{T} & 0 & 0
\end{array}\right]^{-1}\left[\begin{array}{l}
0 \\
0 \\
\varepsilon
\end{array}\right]
$$

em que

$$
\begin{aligned}
& {\left[\begin{array}{c}
x(\varepsilon)-x^{*} \\
\mu(\varepsilon)-\mu^{*} \\
\lambda(\varepsilon)-\lambda^{*}
\end{array}\right]=\left[\begin{array}{c}
\Delta x \\
\Delta \mu \\
\Delta \lambda
\end{array}\right] ;} \\
& \nabla^{2} L^{*}=\nabla_{x x}^{2} L\left(x^{*}, \lambda^{*}, \mu^{*}, \varepsilon\right),
\end{aligned}
$$


sendo:

$$
\begin{aligned}
& \nabla^{2} L^{*}=\nabla_{x x}^{2} f\left(x^{*}\right)+\sum_{i=l}^{m} \lambda_{i} \nabla_{x x}^{2} g_{i}\left(x^{*}\right)+\sum_{j=l}^{r} \mu_{j} \nabla_{x x}^{2} h_{j}\left(x^{*}\right) ; \quad M^{*}=\left[\begin{array}{ccc}
\mu_{l}^{*} & \cdots & 0 \\
\vdots & \ddots & \vdots \\
0 & \cdots & \mu_{r}^{*}
\end{array}\right] ; \\
& D_{H}{ }^{*}=\left[\begin{array}{ccc}
h_{l}\left(x^{*}\right) & \cdots & 0 \\
\vdots & \ddots & \vdots \\
0 & \cdots & h_{r}\left(x^{*}\right)
\end{array}\right] ; G^{*}=\left[\nabla g_{I}\left(x^{*}\right), \ldots, \nabla g_{m}\left(x^{*}\right)\right] ; H^{*}=\left[\nabla h_{l}\left(x^{*}\right), \ldots, \nabla h_{r}\left(x^{*}\right)\right] \\
& \text { e } \varepsilon=\left[\begin{array}{l}
\varepsilon_{l} \\
\vdots \\
\varepsilon_{m}
\end{array}\right] .
\end{aligned}
$$

O sistema matricial (8) é utilizado para obter o novo estado da rede quando uma perturbação $\varepsilon$ nas restrições de igualdade é imposta.

\subsection{Algoritmo}

Um algoritmo proposto para resolver o problema de FPO perturbado consiste dos seguintes passos:

i. Tendo a solução ótima do problema de FPO sem perturbação $(\varepsilon=0)$, impor as perturbações no problema $(\varepsilon \neq 0)$.

ii. Usar (8) para encontrar o novo estado da rede.

iii. Verificar as condições de KKT. Se estiverem satisfeitas dentro de uma determinada tolerância o problema está resolvido, senão obter a solução ótima perturbada usando um programa de FPO.

\section{Testes e Resultados}

Para comprovar a eficiência da abordagem proposta, faremos uma aplicação a um exemplo didático e ao problema de FPO. A implementação computacional foi realizada em linguagem FORTRAN.

\subsection{Exemplo didático}

Para exemplificar a técnica descrita, apresentaremos dois testes. O primeiro com uma perturbação de $2 \%$ e o segundo com $10 \%$ na restrição de igualdade do problema (9). Depois de realizadas as perturbações no problema, estimaremos as novas soluções. Considere o seguinte problema perturbado:

$$
\begin{array}{ll}
\min & x_{1}^{2}+x_{2}^{2} \\
\text { s.a } & x_{1}+2 x_{2}=1+\varepsilon_{1} \\
& x_{1}^{2}-x_{2} \geq 0
\end{array}
$$


Como descrito no algoritmo, seção 3, resolvemos o problema sem perturbação para encontrar o ponto ótimo inicial, '*'. Após encontrado o ponto ótimo do problema aplica-se a técnica de análise de sensibilidade para estimar o novo ponto ótimo, “o”, quando $\varepsilon_{1} \neq 0$. Para validar a técnica, compararemos o ponto estimado com o ponto ótimo após a perturbação.

Ao problema (9), onde todas as restrições estão ativas, podemos associar a seguinte função Lagrangiana:

$$
L=\left(x_{1}^{2}+x_{2}^{2}\right)+\lambda_{1}\left(x_{1}+2 x_{2}-1-\varepsilon_{1}\right)+\mu_{1}\left(x_{1}^{2}-x_{2}\right)
$$

A solução do problema (9) pode ser facilmente encontrada. Aplicando as condições de otimalidade, temos:

$$
\nabla L\left(x_{1}, x_{2}, \lambda_{1}, \mu_{1}\right)=0 .
$$

Resolvendo o problema para $\varepsilon_{1}=0$, temos a solução para o caso base representada na Tabela 1 e visualizada na Figura 1. Os círculos correspondem às curvas de nível da função objetivo, a parábola e a reta às restrições do problema, como pode ser visto na Figura 1.

Tabela 1 - Solução ótima do problema para $\varepsilon_{1}=0$.

\begin{tabular}{|c|c|c|c|c|c|}
\hline$\varepsilon_{1}=0$ & $x_{1}$ & $x_{2}$ & $\lambda_{1}$ & $\mu_{1}$ & $F(x)$ \\
\hline '* & 0,5000 & 0,2500 & $-0,5000$ & $-0,5000$ & 0,3125 \\
\hline
\end{tabular}

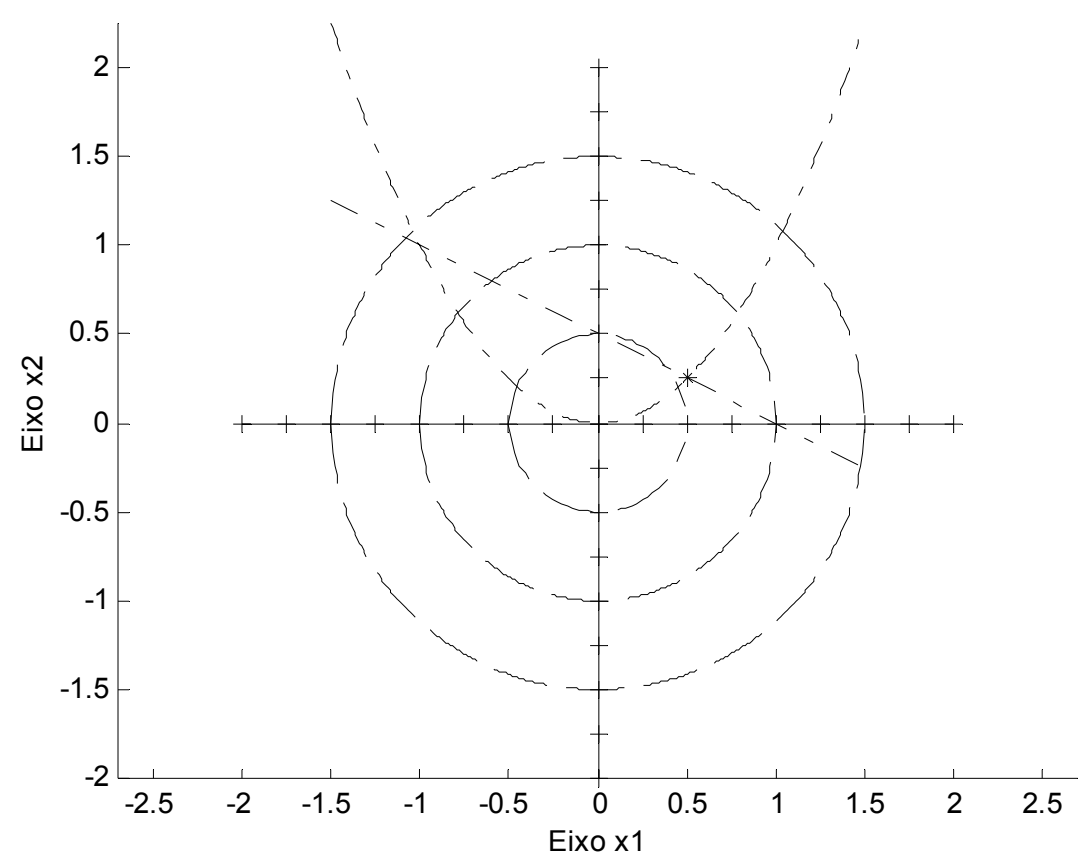

Figura 1 - Representação gráfica da solução ótima do problema para $\varepsilon_{1}=0$. 
Aplicaremos uma perturbação de $\varepsilon_{1}=0,02$ e $\varepsilon_{1}=0,10$ corresponde as variações de $2 \%$ e $10 \%$, respectivamente, nos recursos da restrição de igualdade. Para cada caso compararemos a solução obtida pela técnica de sensibilidade com um método de tipo lagrangiano. As Tabelas 2 e 3 mostram os pontos obtidos via análise sensibilidade "o", e o ponto exato, “*”, para $\varepsilon_{1}=0,02$ e $\varepsilon_{1}=0,10$, respectivamente, os quais podem ser visualizados nas Figuras 2 e 3 respectivamente. A reta contínua, na figura, corresponde à nova posição da restrição de igualdade perturbada.

A aplicação neste exemplo mostra que a técnica de análise de sensibilidade pode ser aplicada em problemas de PNL com bons resultados. Quanto menor a perturbação, melhor o resultado estimado via análise de sensibilidade. A obtenção de novas soluções via análise de sensibilidade ao contrário dos métodos de resolução de problemas PNL, não é iterativa, e não depende da escolha de parâmetros iniciais e de correção, o que faz sua aplicação eficiente. As variáveis duais também são estimadas, fornecendo assim mais informações do problema, como podemos verificar nas colunas 4 e 5 das Tabelas 2 e 3 representados pelas variáveis $\lambda_{1}$ e $\mu_{1}$.

Tabela 2 - Soluções pós-perturbação para $\varepsilon_{1}=0,02$.

\begin{tabular}{|c|c|c|c|c|c|}
\hline$\varepsilon_{1}=0,02$ & $x_{1}$ & $x_{2}$ & $\lambda_{1}$ & $\mu_{1}$ & $F(x)$ \\
\hline "O" & 0,4933 & 0,2433 & $-0,4933$ & $-0,5000$ & 0,3025 \\
\hline "*” & 0,4933 & 0,2433 & $-0,4933$ & $-0,5000$ & 0,3025 \\
\hline
\end{tabular}

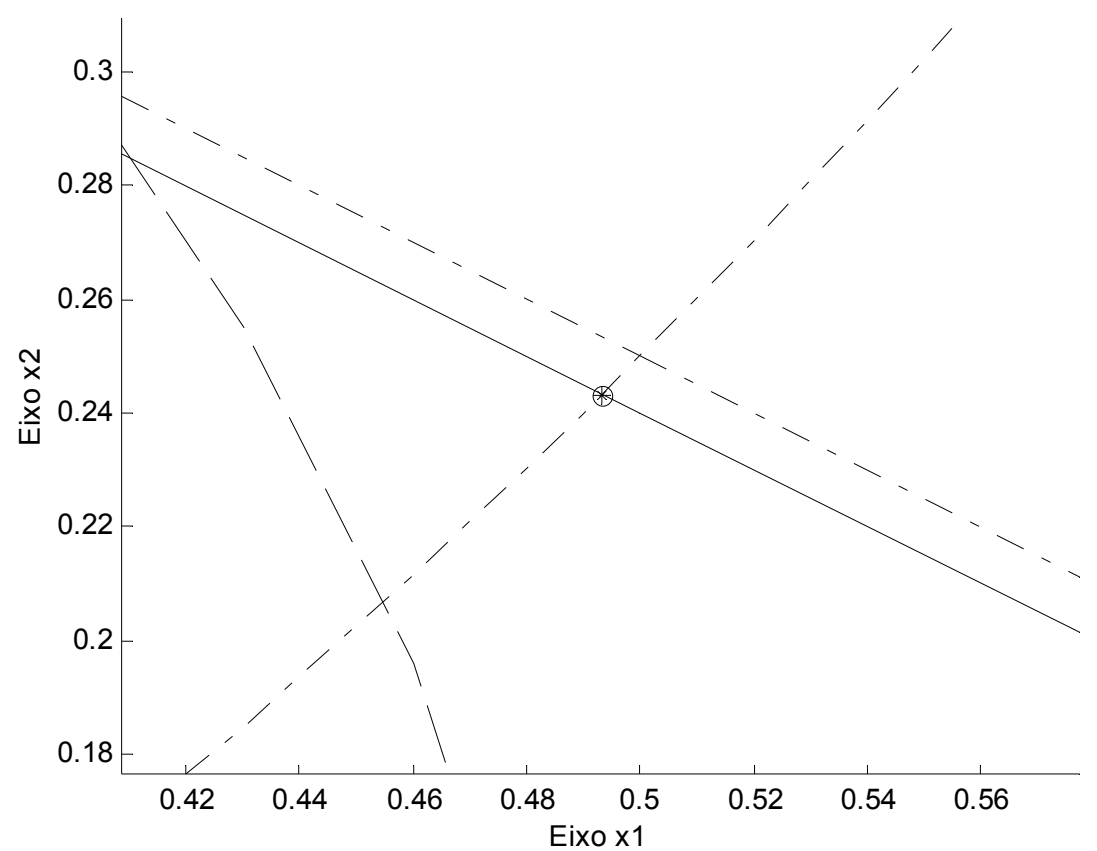

Figura 2 - Representação gráfica da solução para $\varepsilon_{1}=0,02$. 
Tabela 3 - Soluções pós-perturbação para $\varepsilon_{1}=0,10$.

\begin{tabular}{|c|c|c|c|c|c|}
\hline$\varepsilon_{1}=0,10$ & $x_{1}$ & $x_{2}$ & $\lambda_{1}$ & $\mu_{1}$ & $F(x)$ \\
\hline "O" & 0,4659 & 0,2171 & $-0,4666$ & $-0,4992$ & 0,2649 \\
\hline “*” & 0,4667 & 0,2167 & $-0,4667$ & 0,5000 & 0,2647 \\
\hline
\end{tabular}

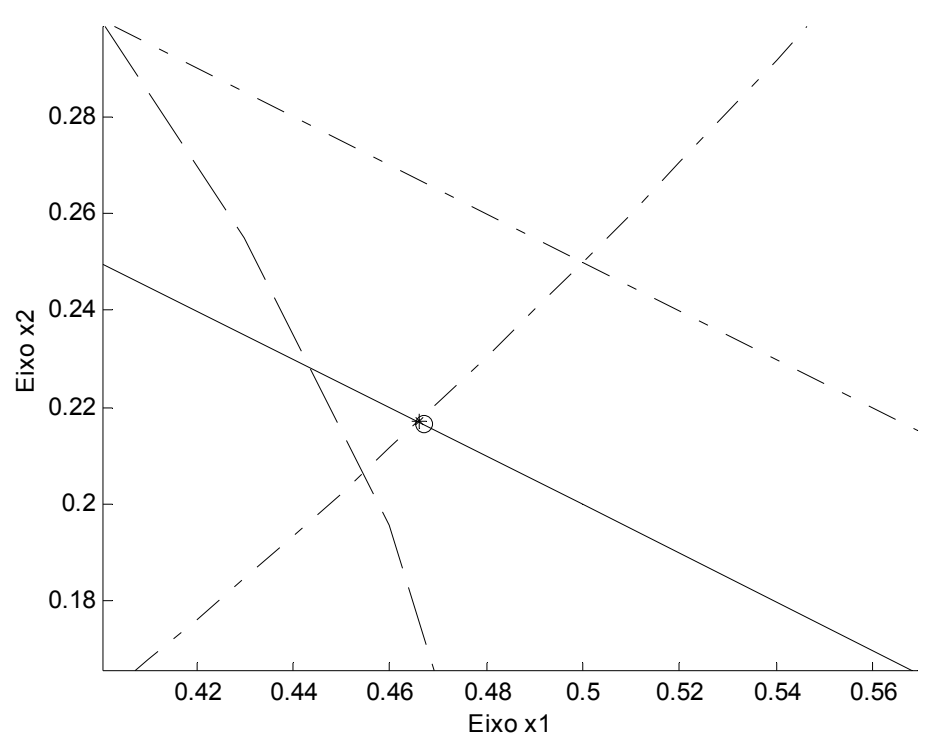

Figura 3 - Representação gráfica da solução para $\varepsilon_{1}=0,10$.

\subsection{Aplicação ao problema de fluxo de potência ótimo}

Três estudos serão analisados utilizando a técnica de análise de sensibilidade ao problema de FPO referente aos sistemas: IEEE 30, IEEE 300 e o SUL-SUDESTE brasileiro de 787 barras. Os dados dos sistemas do IEEE encontram-se no sitio http://www.ee.washington.edu/research/pstca/. A função objetivo do problema de FPO utilizada foi perdas de potência ativa na transmissão, que é uma função não-linear, não convexa e não separável, que segundo Monticelli (1992) torna o problema um dos mais difíceis de resolver.

\subsubsection{Sistema IEEE 30 barras}

O sistema IEEE 30 barras é composto por: 1 barra de geração (slack), 5 barras de controle de potência reativa com geração de potência ativa fixa, 24 barras de carga, 37 linhas de transmissão, 4 transformadores com tap variáveis. O problema de FPO associado ao sistema IEEE 30 barras é composto por 53 restrições de igualdade, 5 restrições canalizadas de potência reativa, quatro restrições canalizadas de tap e 30 restrições canalizadas de tensão. 
Neste sistema realizamos um acréscimo de carga de 5\% em todas as barras de carga. A Tabela 4 mostra as variáveis de magnitude de tensão, $V$, por unidade e ângulo de fase, $\theta$, em graus, das barras do sistema antes da perturbação e após a perturbação estimada via análise de sensibilidade.

Tabela 4 - Soluções do sistema IEEE 30 barras.

\begin{tabular}{|c|c|c|c|c|}
\hline \multirow{2}{*}{ Barras k } & \multicolumn{2}{|c|}{ Caso Base } & \multicolumn{2}{|c|}{ Após a Perturbação } \\
\hline & $V_{k}$ (p.u.) & $\theta_{k}$ (graus) & $V_{k}$ (p.u.) & $\theta_{k}$ (graus) \\
\hline 1 & 1,1000 & 0,0000 & 1,1000 & 0,0000 \\
\hline 2 & 1,0819 & $-4,9276$ & 1,0802 & $-5,0554$ \\
\hline 3 & 1,0639 & $-6,9875$ & 1,0622 & $-7,21608$ \\
\hline 4 & 1,0553 & $-8,6113$ & 1,0533 & $-8,89574$ \\
\hline 5 & 1,0469 & $-13,0530$ & 1,0447 & $-13,3225$ \\
\hline 6 & 1,0505 & $-10,2456$ & 1,0484 & $-10,5744$ \\
\hline 7 & 1,0416 & $-11,8931$ & 1,0389 & $-12,224$ \\
\hline 8 & 1,0513 & $-10,9565$ & 1,0486 & $-11,285$ \\
\hline 9 & 1,0530 & $-13,3326$ & 1,0564 & $-13,8379$ \\
\hline 10 & 1,0457 & $-14,9722$ & 1,0461 & $-15,5671$ \\
\hline 11 & 1,0716 & $-13,3326$ & 1,0864 & $-13,8379$ \\
\hline 12 & 1,0504 & $-14,2300$ & 1,0498 & $-14,7858$ \\
\hline 13 & 1,0744 & $-14,2300$ & 1,0756 & $-14,7858$ \\
\hline 14 & 1,0361 & $-15,0821$ & 1,0349 & $-15,6799$ \\
\hline 15 & 1,0328 & $-15,2079$ & 1,0315 & $-15,8121$ \\
\hline 16 & 1,0403 & $-14,8483$ & 1,0397 & $-15,4387$ \\
\hline 17 & 1,0388 & $-15,1422$ & 1,0387 & $-15,7452$ \\
\hline 18 & 1,0252 & $-15,8273$ & 1,0239 & $-16,4646$ \\
\hline 19 & 1,0238 & $-16,0198$ & 1,0226 & $-16,6676$ \\
\hline 20 & 1,0284 & $-15,8064$ & 1,0276 & $-16,4433$ \\
\hline 21 & 1,0332 & $-15,4058$ & 1,0328 & $-16,0207$ \\
\hline 22 & 1,0336 & $-15,3938$ & 1,0331 & $-16,0075$ \\
\hline 23 & 1,0236 & $-15,5693$ & 1,0218 & $-16,1866$ \\
\hline 24 & 1,0204 & $-15,7609$ & 1,0185 & $-16,3805$ \\
\hline 25 & 1,0193 & $-15,3809$ & 1,0164 & $-15,9728$ \\
\hline 26 & 1,0015 & $-15,9485$ & 0,9977 & $-16,5725$ \\
\hline 27 & 1,0271 & $-14,8096$ & 1,0241 & $-15,3665$ \\
\hline 28 & 1,0470 & $-10,8395$ & 1,0446 & $-11,19$ \\
\hline 29 & 1,0071 & $-15,9880$ & 1,0030 & $-16,6119$ \\
\hline 30 & 0,9949 & $-16,9345$ & 0,9902 & $-17,6141$ \\
\hline Perdas & \multicolumn{2}{|c|}{$16,27 \mathrm{MW}$} & \multicolumn{2}{|c|}{$17,22 \mathrm{MW}$} \\
\hline
\end{tabular}


Ao analisarmos os resultados encontrados na Tabela 4, observarmos que as magnitudes de tensão permaneceram dentro dos seus limites que eram de 0,95 a 1,10 p.u. Os maiores erros nas equações de desigualdade ocorreram nas barras 6 e 3 respectivamente para as potências ativas e reativas, os quais ficaram na ordem de $10^{-4}$ p.u. As perdas de potência ativa na transmissão passaram de 16,27 MW para 17,22 MW. A geração de potência ativa na barras slack teve um acréscimo de 7,8 MW após a perturbação. É importante notar que a tensão na barra 1 estava no limite superior, e mesmo após a perturbação no sistema o limite foi satisfeito. Isso ocorreu devido ao multiplicador de Lagrange associado à restrição da tensão na barra estar ativo na solução do caso base.

\subsubsection{Sistema IEEE 300 barras}

O sistema IEEE 300 barras é composto por: 1 barra de geração (slack), 68 barras de controle de potência reativa com geração de potência ativa fixa, 231 barras de carga, 411 linhas de transmissão e 107 transformadores com tap variáveis. O problema de FPO associado a este sistema é composto por 530 restrições de igualdade, 68 restrições canalizadas de potência reativa, 107 restrições canalizadas de tap e 300 restrições canalizadas de tensão.

A este sistema realizamos um acréscimo de carga de 5\% nas barras de carga 49, 51, 52 e 55 mantendo o fator de potência constante. Utilizado a técnica de análise de sensibilidade foi estimada a solução após a perturbação. Neste teste foi analisada a relação de sensibilidade do acréscimo de carga destas barras em relação à injeção de potência reativa nas barras de geração. Após a aplicação da técnica aqui apresentada, observamos que as injeções de potência reativa permaneceram dentro dos seus limites. Os maiores erros nas equações de igualdade ocorreram na barra de carga 526. O erro para potência ativa foi menor que $9 \times 10^{-4}$ p.u. e o erro para potência reativa, menor que $2 \times 10^{-4} \mathrm{p}$.u. As perdas no sistema passaram de 408,31 MW para 410,06 MW após o acréscimo de carga. A barra slack teve um acréscimo de 9,7 MW em sua geração após a perturbação. A Tabela 5 mostra a variação da injeção de potência reativa com o aumento de carga no sistema. As maiores variações de injeção de potência reativa ocorreram na barra 7049 (slack) e nas barras de controle de reativos 119 e 143 .

Tabela 5 - Análise de sensibilidade da injeção de potência reativa no sistema em relação ao aumento de carga de 5\% nas barras de carga 49, 51, 52 e 55 .

\begin{tabular}{|c|c|c|c|}
\hline Barra k & $\begin{array}{c}\text { Reativos Antes da } \\
\text { Perturbação no Ponto } \\
\text { Ótimo (MVAr) }\end{array}$ & $\begin{array}{c}\text { Reativos Após a } \\
\text { Perturbação (MVAr) }\end{array}$ & $\begin{array}{c}\text { Variação da Injeção } \\
\text { de Reativos (MVAr) }\end{array}$ \\
\hline 7049 & 72,8 & 85,8 & 13,0 \\
\hline 8 & $-59,9$ & $-59,2$ & 0,7 \\
\hline 10 & 56,6 & 57,7 & 1,1 \\
\hline 20 & 102,9 & 101,6 & $-1,3$ \\
\hline 63 & 52,0 & 49,0 & $-3,0$ \\
\hline 76 & 143,3 & 143,7 & 0,4 \\
\hline 84 & 120,1 & 120,2 & 0,1 \\
\hline 91 & 81,5 & 81,0 & $-0,5$ \\
\hline 92 & 81,8 & 81,6 & $-0,2$ \\
\hline
\end{tabular}


Belati, Souza, Baptista \& Costa - Determinação da solução ótima do problema de fluxo de potência ótimo via análise de sensibilidade

\begin{tabular}{|c|c|c|c|}
\hline 98 & $-60,4$ & $-59,8$ & 0,6 \\
\hline 108 & 3,2 & 3,9 & 0,7 \\
\hline 119 & 981,6 & 987,5 & 5,9 \\
\hline 124 & 98,2 & 97,7 & $-0,5$ \\
\hline 125 & 191,5 & 191 & $-0,5$ \\
\hline 138 & 192,4 & 192,5 & 0,1 \\
\hline 141 & 72,3 & 72,4 & 0,1 \\
\hline 143 & 267,9 & 276,1 & 8,2 \\
\hline 146 & 17,7 & 18,2 & 0,5 \\
\hline 147 & $-44,5$ & $-44,2$ & 0,3 \\
\hline 149 & 24,0 & 24,1 & 0,1 \\
\hline 152 & $-48,9$ & $-48,8$ & 0,1 \\
\hline 153 & $-15,1$ & $-14,9$ & 0,2 \\
\hline 156 & $-6,2$ & $-5,9$ & 0,3 \\
\hline 170 & 78,2 & 79,4 & 1,2 \\
\hline 171 & 447,6 & 448,5 & 0,9 \\
\hline 176 & 34,1 & 34,6 & 0,5 \\
\hline 177 & 39,9 & 40,8 & 0,9 \\
\hline 185 & 19,1 & 18,9 & $-0,2$ \\
\hline 186 & 274,6 & 272,3 & $-2,3$ \\
\hline 187 & 215,9 & 214,3 & $-1,6$ \\
\hline 190 & 145,1 & 143,8 & $-1,3$ \\
\hline 191 & 696,0 & 698,7 & 2,7 \\
\hline 198 & 66,2 & 66,2 & 0,0 \\
\hline \multicolumn{2}{|c|}{ Barras mais Sensíveis } & \multicolumn{2}{|c|}{7049,119 e 143} \\
\hline
\end{tabular}

Este teste mostra a sensibilidade da injeção de potência reativa no sistema em relação a um aumento de carga em um determinado grupo de barras. É possível através da técnica determinar a sensibilidade de qualquer variável em relação à variação em uma determinada carga, um grupo de carga e até mesmo em todas as cargas do sistema.

\subsubsection{Sistema SUL-SUDESTE 787 barras}

O sistema SUL-SUDESTE brasileiro é um sistema que envolve as regiões sul e sudeste do Brasil. Ele é composto por: 1 barra de geração (slack), 111 barras de controle de potência reativa, 675 barras de carga, 1309 linhas de transmissão e 204 transformadores. O problema de FPO associado a este sistema é composto por 1461 restrições de igualdade, 111 restrições canalizadas de potência reativa, 787 restrições canalizadas de tensão e 204 restrições canalizadas de tap. A função, perdas na transmissão, a ser otimizada pelo programa de FPO, apresenta 1778 variáveis envolvendo magnitude de tensão, ângulo de fase, e tap de transformador. O programa de FPO utilizou 18 iterações para obter a solução do caso base. 
Neste teste consideramos algumas barras de carga que estão indicadas pela letra 'C' na primeira coluna da Tabela 6, que representam todas as regiões do sistema. Nestas barras foram realizados acréscimos simultâneos de $2,5 \%$ de carga mantendo o fator de potência constante, que representou um acréscimo de 39,85 MW de potência ativa distribuídas no sistema. Utilizado a técnica de análise de sensibilidade foi estimada a solução após a perturbação e, ressalta-se que todas as restrições de desigualdade foram satisfeitas. O maior erro das restrições de igualdade foi de $3 \times 10^{-3} \mathrm{p} . \mathrm{u}$.

As perdas no sistema passaram de 1787,31 MW para 1788,54 MW após o acréscimo de carga. A barra slack teve um acréscimo de 41,09 MW em sua geração. A Tabela 6 mostra as variáveis de magnitude de tensão, $V$, por unidade e ângulo de fase, $\theta$, em graus, para o conjunto de barras escolhidas, onde destacamos algumas barras de geração indicadas pela letra ' $G$ ' e as barras de carga perturbadas. Na tabela também é destacada a potência gerada e consumida destas barras no caso base.

Tabela 6 - Sistema SUL-SUDESTE.

\begin{tabular}{|c|c|c|c|c|c|c|}
\hline \multirow[t]{2}{*}{ Barras } & \multicolumn{2}{|c|}{$\begin{array}{c}\text { Potência nas Barras } \\
\text { Caso Base }\end{array}$} & \multicolumn{2}{|c|}{ Caso Base } & \multicolumn{2}{|c|}{ Após a Perturbação } \\
\hline & MW & MVAr & $V_{k}$ (p.u.) & $\theta_{k}$ (graus) & $V_{k}$ (p.u.) & $\theta_{k}$ (graus) \\
\hline Furnas - G & 957,11 & 714,53 & 1,0935 & $-9,0000$ & 1,0937 & $-9,0000$ \\
\hline G. B. M. R. Neto - G & 1506,00 & 42,70 & 1,0297 & 33,1040 & 1,0299 & 32,9494 \\
\hline Salto Santiago - G & 1100,00 & $-5,41$ & 1,0348 & 35,8789 & 1,0348 & 35,7301 \\
\hline Itaipu - G & 5500,00 & 705,00 & 1,0406 & 34,4578 & 1,0404 & 34,3374 \\
\hline Marimbondo - G & 1250,00 & $-30,70$ & 1,0646 & 12,6166 & 1,0648 & 12,5861 \\
\hline Ilha Solteira - G & 2200,00 & $-394,00$ & 1,0872 & 14,1680 & 1,0875 & 14,0303 \\
\hline Porto Primavera - G & 1100,00 & $-192,00$ & 1,0522 & 23,0054 & 1,0522 & 22,8681 \\
\hline São Simão - G & 1400,00 & 121,00 & 1,0221 & 22,6332 & 1,0235 & 22,6107 \\
\hline Pelotas - C & 133,00 & 43,70 & 1,0044 & 14,9084 & 1,0094 & 14,3769 \\
\hline Tubarão - C & 13,40 & 4,50 & 1,0178 & 12,9614 & 1,0176 & 12,7789 \\
\hline Canoinhas - C & 41,50 & 18,60 & 0,9945 & 8,4701 & 0,9937 & 8,2550 \\
\hline Araraquara - $\mathrm{C}$ & 506,00 & 137,00 & 1,0240 & $-4,3930$ & 1,0237 & $-4,5595$ \\
\hline S. J. R. Preto - C & 462,00 & 143,00 & 0,9867 & $-7,7673$ & 0,9857 & $-8,0014$ \\
\hline Taubaté - C & 348,00 & 0,68 & 1,0014 & $-24,6491$ & 1,0012 & $-24,8926$ \\
\hline \multirow[t]{2}{*}{ Pimenta - C } & 90,10 & 18,00 & 0,9964 & $-18,5532$ & 0,9517 & $-39,4241$ \\
\hline & \multicolumn{2}{|c|}{ Perdas } & \multicolumn{2}{|c|}{ 1787,31 MW } & \multicolumn{2}{|c|}{$1788,54 \mathrm{MW}$} \\
\hline
\end{tabular}

Este teste comprova que a técnica de análise de sensibilidade pode ser aplicada a sistemas reais para pequenas perturbações, sendo que o custo computacional se limita apenas na execução de um FPO para o caso base. 


\section{Conclusões}

O artigo apresenta uma metodologia para resolução do problema de FPO perturbado que pode ser estendida para outros problemas de PNL. A metodologia consiste, primeiramente, na obtenção de uma solução ótima inicial para o caso base via o FPO, e na utilização da técnica de análise de sensibilidade para estimar novas soluções depois de ocorridas pequenas perturbações na demanda do sistema. Essas perturbações são um acréscimo ou um corte de carga em uma ou mais barras do sistema. Com a aplicação da técnica foi possível obter novos pontos de operação para o sistema, sem a necessidade de se reprocessar o programa de FPO.

As principais contribuições do método em comparação com os programas de FPO são: algoritmo de resolução não iterativo; e não necessidade de se utilizarem parâmetros iniciais e de correção, como os de barreira e de penalidade, utilizados nos programas de FPO convencionais.

Os resultados numéricos apresentados neste trabalho evidenciam o potencial do método apresentado, para a resolução de problemas de FPO perturbado. Os testes demonstraram que a metodologia pode ser aplicada após perturbações nas restrições de igualdades, sendo que quanto maior os sistemas, menores serão as perturbações admissíveis pela técnica de sensibilidade empregada.

A técnica de análise de sensibilidade também pode ser usada para estimar soluções onde os algoritmos de FPO não conseguem êxito, como por exemplo, em alguns casos de estudos da expansão do SEP.

\section{Agradecimentos}

À FAPESP (Fundação de Amparo à Pesquisa do Estado de São Paulo) pelo apoio financeiro.

\section{Referências Bibliográficas}

(1) Aumuller, C. \& Sara, T.K. (2002). Analysis and Assessment of Large Scale Power System Voltage Stability by a Novel Sensitivity Based Method. In: Power Engineering Society Summer Meeting, IEEE, 3, 1621-1626.

(2) Baptista, E.C.; Belati, E.A. \& Costa, G.R.M. (2004). Um Método Primal-Dual Aplicado na Resolução do Problema de Fluxo de Potência Ótimo. Pesquisa Operacional, 24, 215-226.

(3) Belhadj, C. et al. (1996). Voltage Stability Modeling and Real-Time Monitoring Using Expert System for Operation Assistance. IEEE Transactions on Power Systems, 11(2), 1037-1042.

(4) Costa, G.R.M. (1997). Optimal Reactive Dispatch Through Primal-dual Method. IEEE Transactions on Power Systems, 12(2), 669-674.

(5) Dillon, T.S. (1981). Rescheduling, Constraints Participation Factors and Parameter Sensibility in the Optimal Power Flow Problem. IEEE Transactions on PowerApparatus and Systems, 100(5), 2628-2634. 
(6) Fiacco, A.V. (1976). Sensitivity Analysis for Nonlinear Programming Using Penalty Methods. Mathematical Programming, 10(3), 278-311.

(7) Granville, S. (1994). Optimal Reactive Dispatch Through Interior Point Methods. IEEE Transactions on Power Systems, 9, 136-146.

(8) Gribik, P.R.; Shirmohammdi, D.; Hao, S. \& Thomas, C.L. (1990). Optimal Power Flow Sensitivity Analysis. IEEE Transactions on Power Systems, 5(3), 969-976.

(9) Iba, K. et al. (1998). Pratical Reactive Power Allocation/Operation Planning using Successive Linear Programming. IEEE Transactions on Power Systems, 3(2), 558-567.

(10) Kishore, A. \& Hill, E.F. (1971). Static Optimization of Reactive Power Sources by Use of Sensitivity Parameters. IEEE Transactions on Power Systems, 90(3), 1166-1173.

(11) Monticelli, J.A. \& Liu, W.H.E. (1992). Adaptative Movement Penalty Method for Newton Optimal Power Flow. IEEE Transactions on Power Systems, 7(1), 334-341.

(12) Park, Y.M. \& Lee, K.H. (1997). Application of Expert System to Power System Restoration in Sub-Control Center. IEEE Transactions on Power Systems, 12(2), 629-635. 